\title{
Análisis e interpretación de la sismicidad local en Veracruz para la generación de boletines del OSV
}

\section{Analysis and interpretation of local seismicity in Veracruz for the generation of OSV bulletins}

Francisco Córdoba-Montiel ${ }^{\mathrm{a}}$ - Xyoli Pérez Campos ${ }^{\mathrm{b}}$-Katrin Sieron ${ }^{\mathrm{c}}$-Sergio Francisco Juárez Cerrillo ${ }^{d}-$ Grupo de Trabajo del Servicio Sismológico Nacional

a Observatorio Sismológico y Vulcanológico, Centro de Ciencias de la Tierra, Universidad Veracruzana, Xalapa México. Contacto: fcordoba@uv.mx.

${ }^{\text {b }}$ Departamento de Sismología, Instituto de Geofísica, Universidad Nacional Autónoma de México, Ciudad de México, México. Contacto: xyoli@geofisica.unam.mx

c Observatorio Sismológico y Vulcanológico, Centro de Ciencias de la Tierra, Universidad Veracruzana, Xalapa, México. Contacto: ksieron@uv.mx.

d Facultad de Estadística e Informática, Universidad Veracruzana, Xalapa, México. Contacto: sejuarez@uv.mx

Recibido: 13 de septiembre de 2020

Aceptado: 15 de octubre de 2020

RESUMEN: A partir de la instalación y puesta en operación de la Red Sísmica de Banda Ancha de Veracruz (RSBAV) en 2013 y su integración a la red de instrumentos del Servicio Sismológico Nacional (SSN), una mejor detección, observación y comprensión de la sismicidad local y regional en el oriente y sur de México ha sido posible. En consecuencia, con los datos obtenidos se contribuye a la detección oportuna de sismos en la región, que, como fuente oficial nacional, reporta el SSN. Lo anterior ha generado una mayor cantidad de datos que es necesario describir y comprender de acuerdo con el contexto sísmico y tectónico local y regional. Motivado por lo anterior, la Universidad Veracruzana, a través del Observatorio 
Sismológico y Vulcanológico de Veracruz, ha incorporado en su página web una sección donde se presentará semestralmente un boletín con una revisión cuantitativa y cualitativa de la actividad sísmica en el territorio veracruzano que reporta el SSN. La finalidad es coadyuvar a un mejor entendimiento y una visión realista de los fenómenos sísmicos, así como caracterizar la sismicidad base de Veracruz. En este trabajo se presenta una propuesta del formato del boletín con los datos correspondientes al primer semestre de 2020, describiéndose los elementos que deben ser considerados para futuras consultas y referencias.

Palabras clave: Placas tectónicas; sismicidad base; sismos intraplaca e interplaca; magnitud; epicentro; subducción.

ABSTRACT: From the installation and start-up of the Veracruz Seismic Broadband Network (RSBAV) in 2013 and its integration to the instrument network of the National Seismological Service (SSN), a better detection, observation and understanding of local seismicity and regional in the east and south of Mexico has been possible. Consequently, the data obtained contributes to the timely detection of earthquakes in the region, which as an official national source, reports the SSN. This has generated a greater amount of data that needs to be described and understood according to the local and regional seismic and tectonic context. Motivated by the above, the Universidad Veracruzana, through the Seismological and Volcanological Observatory of Veracruz, has incorporated a section on its website where a bulletin will be presented every six months with a quantitative and qualitative review of the seismic activity in the Veracruz territory reported by the SSN. The purpose is to contribute to a better understanding and a realistic vision of seismic phenomena, as well as to characterize the base seismicity of Veracruz. In this work, a proposal for the format of the bulletin is presented with the data corresponding to the first semester of 2020, describing the elements that should be considered for future consultations and references.

Keywords: Tectonic Plates; Base Seismicity, Intraplate and Interplate Earthquakes, Magnitude, Epicenter, Subduction.

\section{Introducción}

L os sismos son fenómenos naturales originados por las grandes fuerzas que actúan al interior de la Tierra. Sin embargo, algunos de ellos están asociados a fenómenos locales, como es el caso de la actividad volcánica o incluso los de origen antropogénico, como el 
fracturamiento hidráulico. Los sismos son fenómenos que en México se presentan en su mayoría en las costas del Pacífico (Guerrero, Oaxaca y Chiapas), una zona de "contacto convergente" entre placas donde tiene lugar uno de los procesos tectónicos más importantes. A nivel nacional, el Servicio Sismológico Nacional (SSN) reporta oportuna y permanentemente la actividad sísmica que ocurre en el territorio mexicano.

Un sismo se produce por la ruptura de roca dentro de alguna placa tectónica como consecuencia de los esfuerzos (las fuerzas internas que actúan sobre cierta área) y conlleva el deslizamiento de un bloque sobre otro (de una cierta área conocida como plano de falla). El hipocentro de un sismo es el punto donde inicia una ruptura (el epicentro es su proyección en la superficie), la cual se extiende hasta alcanzar una determinada área cuyo tamaño está relacionado directamente con la magnitud del evento. El proceso de fallamiento produce una liberación repentina de energía que se manifiesta en la generación de ondas sísmicas que causan los movimientos verticales y horizontales conocidos que presentan todos los sismos. La magnitud de un sismo es una medida del tamaño que se determina a partir del registro de sismos. La corteza terrestre, donde ocurren estos eventos, se divide en corteza continental y corteza oceánica, cuyos espesores varían entre 10 y $80 \mathrm{~km}$, siendo más delgada la oceánica. Esta corteza constituye la parte más externa de la Tierra (que por su fragilidad es susceptible a la ruptura, misma que origina los sismos) y conforma los grandes segmentos conocidos como placas tectónicas, que interactúan continuamente entre sí. Los sismos son resultado de los grandes procesos internos del planeta, por lo que siempre han estado presentes y su estudio, contribuye a la prevención y mitigación de daños.

\section{Marco Tectónico y Sismológico}

El contexto tectónico de México tiene su origen en la interacción de cinco placas tectónicas: Norteamérica (donde se asienta la mayor parte del país y la cual consiste mayoritariamente de corteza continental), Pacífico, Rivera, Cocos y Caribe, cuya distribución se ilustra en el mapa de la Figura 1. Derivado de la convección (flujo originado por las altas temperaturas del interior de la Tierra) de material del manto, se produce el movimiento de las placas tectónicas que actúan entre sí por esfuerzos de compresión, extensión y transcurrencia (recuadro de la Figura 1). 


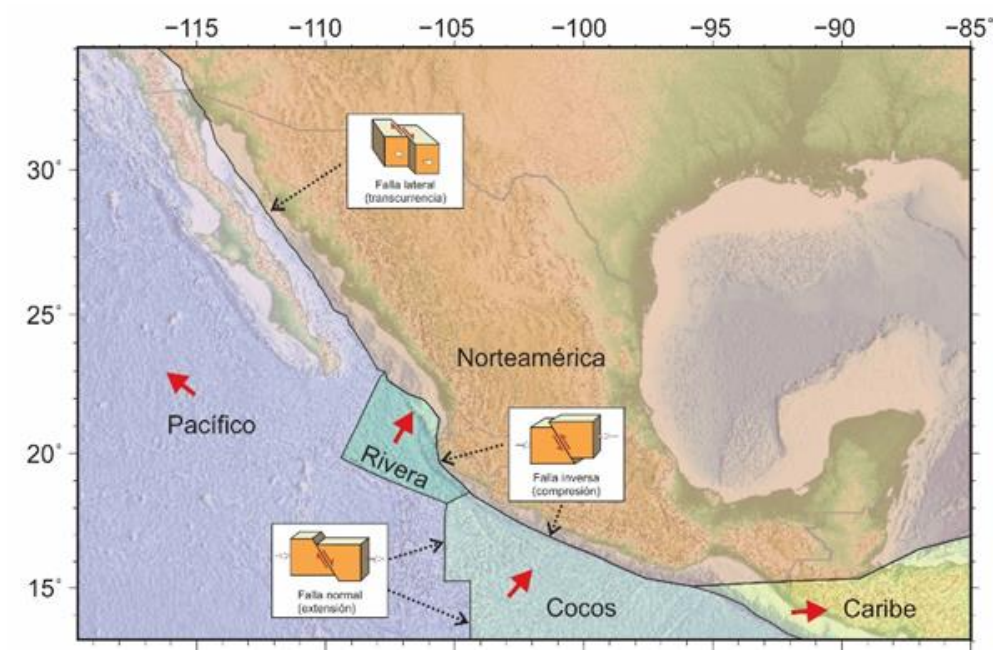

Figura 1. Mapa tectónico de México que ilustra la interacción entre las placas tectónicas que dan origen a la sismicidad del país. En los recuadros se presentan los tres tipos de fallas que se mencionan en el texto (normal, inversa y lateral), ejemplificándose algunos márgenes donde ocurren. Las flechas de color blanco indican la dirección en que actúan los esfuerzos que ocasionan el movimiento de los bloques involucrados.

En la convergencia de placas, donde intervienen esfuerzos de compresión, existe un contacto entre las placas involucradas que se desplazan en dirección opuesta relativa ("chocando" entre ellas). Si se trata de una interacción entre corteza oceánica con corteza continental, la placa más densa (corteza oceánica) se "hunde" o subduce, en tanto que la menos densa (y por lo tanto más boyante, corteza continental) se mantiene a "flote". En México, esto ocurre en las costas del océano Pacífico (a lo largo de la Trinchera Mesoamericana), donde las placas tectónicas cocos y Rivera (corteza oceánica), subducen debajo de la placa Norteamérica (corteza continental). En la zona de contacto, se producen deformaciones como consecuencia de los grandes esfuerzos actuando entre sí, hasta que, al alcanzar el punto de ruptura, se presenta el deslizamiento de los bloques y consecuentemente la liberación repentina de energía (sismo de fallamiento inverso, Figura 1). El proceso de subducción en México es el que da origen a la mayor cantidad de sismos en el país, así como los de mayor magnitud y recurrencia, siendo el evento del 19 de septiembre de 1985 (MS 8.1) el más representativo en su historia reciente.

Una de las consecuencias del proceso de convergencia entre placas tectónicas que ocurre a lo largo de la denominada Trinchera Mesoamericana (Figura 2), fue la formación del arco volcánico continental conocido como Faja Volcánica TransMexicana (FVTM, Figura 2) o Cinturón Volcánico Mexicano (CVM). Esta cadena de aparatos volcánicos atraviesa el país desde Nayarit hasta llegar a la costa del Golfo de México (e.g. Gómez Tuena et al., 2005 y 
Ferrari et al., 2012). El vulcanismo, igual que parte de la sismicidad, es consecuencia directa de la subducción de las placas Rivera y Cocos debajo de la Norteamericana, conllevando procesos de fusión de las placas subducidas, y la formación de magmas en consecuencia.

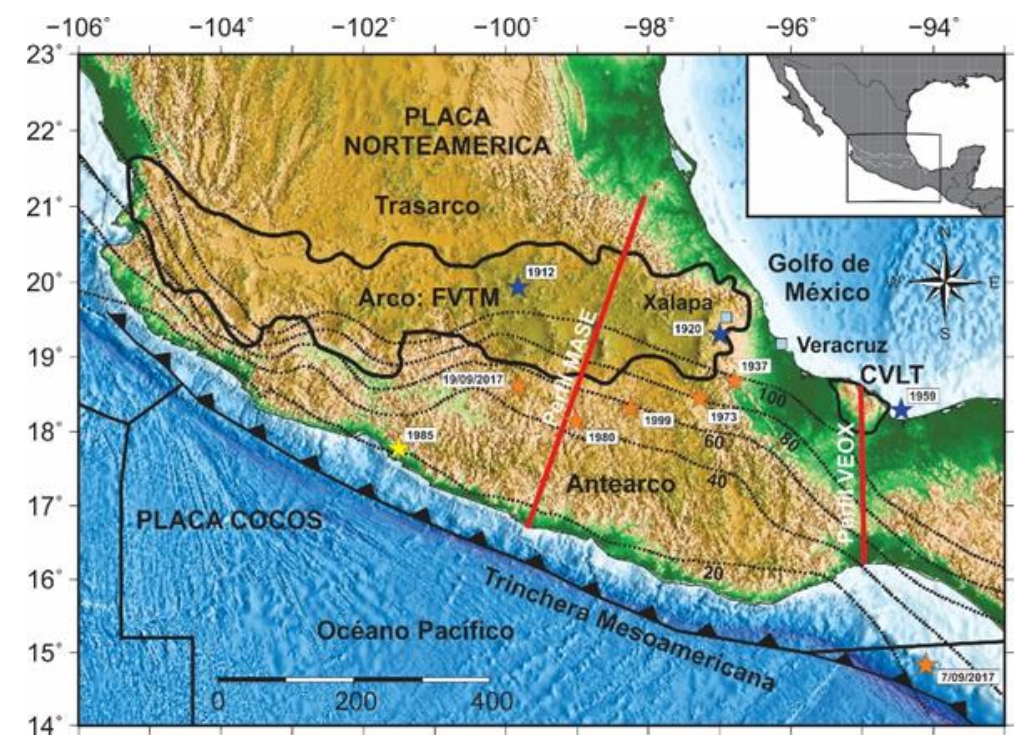

Figura 2. Contexto sismotéctonico del oriente de México, donde destaca la presencia de la Faja Volcánica TransMexicana (FVTM, sector oriental), el Campo Volcánico Los Tuxtlas (CVLT), las isoprofundidades de la placa subducida (Pardo y Suárez, 1995) y la sismicidad histórica del área de interés del presente trabajo. Los cuadrados en color azul representan la ubicación de las ciudades de Xalapa y Veracruz. Las estrellas corresponden a epicentros de algunos eventos importantes en el pasado, que de acuerdo con su color, indican su tipo: en azul corticales, naranja intraplaca y en amarillo, el del sismo interplaca de 1985.

En el movimiento de extensión entre placas Tectónicas, los esfuerzos que actúan en cada una las placas son divergentes (Figura 1), de tal forma que las fuerzas que intervienen "jalan" en dirección opuesta hasta alcanzar el punto en el que se produce la ruptura y el consecuente deslizamiento de los bloques (fallamiento normal, Figura 1). Un ejemplo de este proceso ocurre en medio de los océanos del mundo, a lo largo de las dorsales oceánicas y tiene como efecto la creación de corteza nueva, dado que la separación que se produce permite el ascenso de magma.

Por último, cuando existen esfuerzos transcurrentes entre las placas tectónicas, se produce un desplazamiento lateral entre ellas (Figura 1), por lo que no existe consumo de corteza (como en la subducción), ni generación de corteza nueva como en el caso de los esfuerzos de extensión. En México, este tipo de movimiento se da entra las placas Norteamérica y Pacifico (Figura 1) y ha producido sismos importantes en el país. Uno de los casos en el mundo, más 
conocidos de este tipo de límites entre dos placas, es la zona de la Falla de San Andrés (EE. UU.).

Los sismos no se producen únicamente en las zonas donde existe contacto de placas, sino que además se pueden originar dentro de una misma placa y se les denomina sismos intraplaca. Aunque los mayores esfuerzos (y en consecuencia las mayores deformaciones) se generan en las zonas de contacto entre placas antes descritas, el efecto indirecto de esta interacción sobre las placas involucradas, aunado a los esfuerzos que imponen las condiciones de la geología local, influyen de manera determinante en la generación de este otro tipo de eventos. Los mecanismos que producen el fallamiento de estos sismos son los mismos presentes en los eventos interplaca: inverso, normal y lateral.

Los sismos intraplaca pueden clasificarse en corticales e intraplaca: los corticales son aquellos que ocurren en la corteza continental, que en México suceden principalmente en la placa Norteamérica (Figuras 1 y 3), donde se asienta la mayor parte del territorio del país. Cuando estos eventos son poco profundos (generalmente $\mathrm{H}<15 \mathrm{~km}$ ), se les refiere como sismos someros. Aunque no llegan a ser de gran magnitud como los sismos interplaca, tienen un potencial de daños elevado a causa de su poca profundidad y la corta distancia a centros poblacionales importantes. En el siglo pasado, el sismo de Acambay de 1912 (Mw 6.7, Singh et al., 2011) y el de Xalapa de 1920 (Mw 6.4, Singh et al., 1984), son dos claros ejemplos.

El otro tipo de sismos intraplaca típico tiene lugar en placas subducidas (corteza oceánica, recuadro de la Figura 1) y que, en el caso de México, corresponden a las placas Rivera y Cocos (Figura 3). A estos eventos se les denomina intraplaca (ver Figura 3) y pueden alcanzar grandes magnitudes a profundidades intermedias o mayores ( $>40 \mathrm{~km}$ ). Entre algunos sismos de este tipo en México (Figura 2) se encuentran el de Orizaba de 1973 (Mw 7.0, H = 82 km), Huajuapan de 1980 (Mw 7.0, H = 82 km), Tehuacán de 1999 (Mw 7.0, H = 61 km) y más recientemente, los ocurridos en septiembre de 2017: el del 7 de septiembre (Mw 8.2, H = 46 km), y el del 19 de septiembre (Mw 7.1, $\mathrm{H}=51 \mathrm{~km}$ ).

\section{Geometría de la subducción}

La geometría de la subducción (la forma en que las placas oceánicas subducen debajo de la continental) es un proceso complejo y variable que se conoce con mejor detalle en México a partir de diversos estudios sismológicos que han empleado metodologías variadas y complementarias con el fin de tener una mejor resolución en sus resultados. Para ilustrar esta 
variabilidad, la Figura 3 (izquierda) sintetiza dos perfiles propuestos por Pacheco y Kostoglodov (1999), correspondientes a los transectos mostrados en el mapa de la Figura 2.
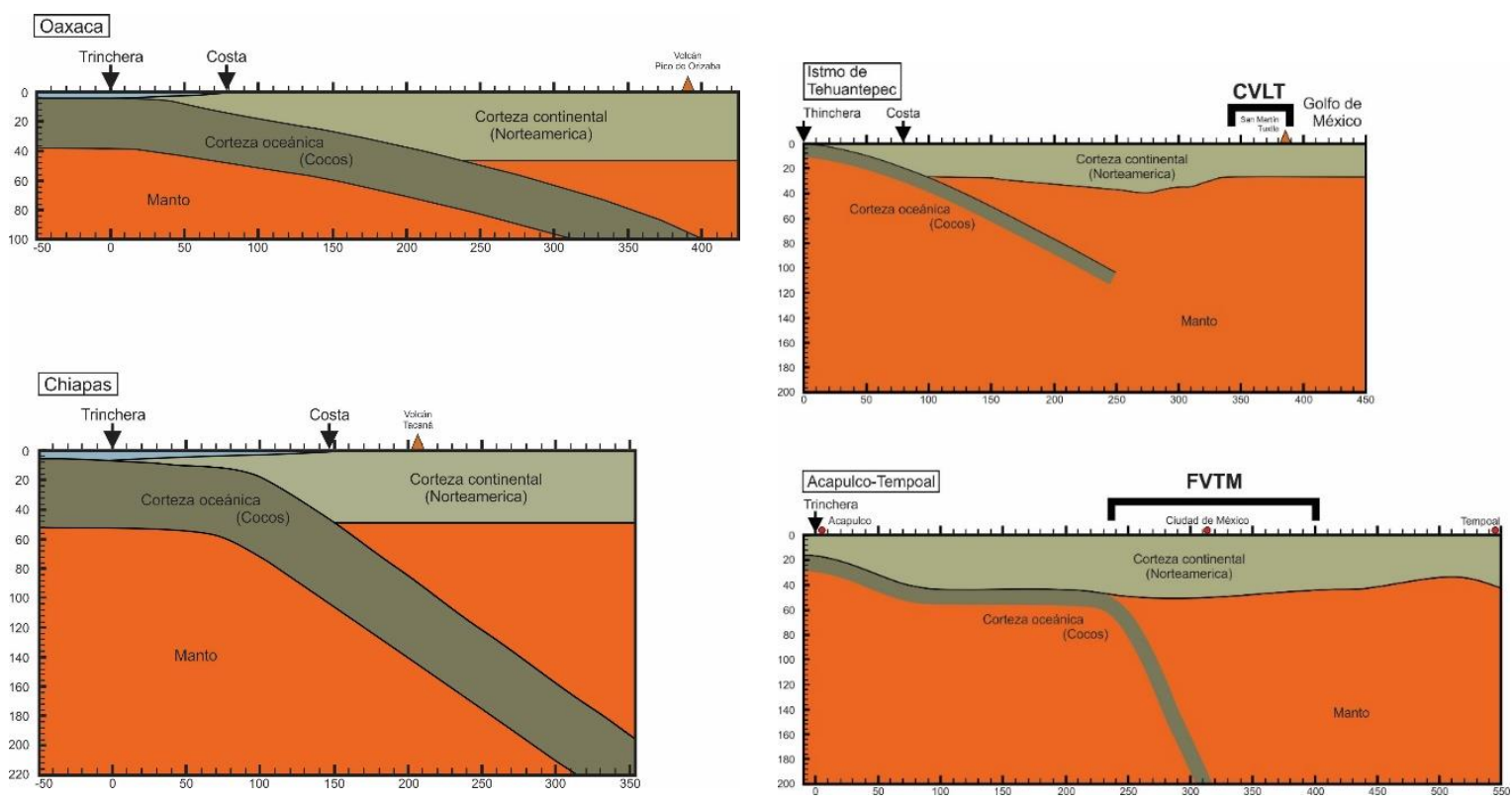

Figura 3. Perfiles de la geometría de subducción mexicana. Izquierda, para los estados de Guerrero, Oaxaca y Chiapas (Modificado de Pacheco y Kostoglodov, 1999). Derecha: modelos simplificados de la corteza y geometría de la subducción para los transectos definido por los arreglos MASE y VEOX (Modificado de Pérez-Campos et al., 2008 y Melgar y Pérez-Campos, 2010).

Recientemente, mediante el empleo de datos de estaciones sísmicas de los proyectos MASE (Mesoamerican Subduction Experiment) y VEOX (Veracruz-Oaxaca), Pérez-Campos et al. (2008) y Melgar y Pérez-Campos (2010), utilizaron la técnica de funciones de receptor para delinear la corteza continental (placa Norteamérica) y la geometría de la placa en subducción (placa Cocos) a lo largo de los transectos definidos por las estaciones de ambos arreglos: Acapulco, Guerrero-Tempoal, Veracruz (MASE); en tanto que para VEOX, el perfil obtenido muestra la geometría desde la trinchera, en el Istmo de Tehuantepec (costas de Oaxaca), hasta las costas de Veracruz, en el Golfo de México, donde se localiza el Campo Volcánico de Los Tuxtlas (CVLT). Para un mejor entendimiento, la Figura 3 (derecha) sintetiza los modelos obtenidos.

Por lo anteriormente expuesto, debe observarse que la sismicidad en una determinada área o región depende de las condiciones definidas dentro de un contexto tectónico amplio, así como de condiciones locales preexistentes. Esto significa entre otras cosas, que para conocer el peligro sísmico de cierta región, se deben estudiar cuidadosamente las condiciones 
Observatorios - Observatorio Sismológico y Vulcanológico

específicas de esa área y evaluar las implicaciones particulares que impone el marco tectónico en el que está inmerso dentro del país.

\section{Caracterización sismotectónica de Veracruz}

Para efectos de análisis, se propone de manera similar a la regionalización sísmica del estado de Veracruz (CFE, 2008), la división de la entidad en tres áreas; norte, centro y sur. Sin embargo, desde el punto de vista tectónico y geológico, se puede reconocer una delimitación basada en los principales rasgos de esta índole que se presentan en el territorio de Veracruz, entre los que sobresalen la FVTM y el CVLT. De acuerdo con Córdoba et al. (2017), luego de revisar sismicidad reportada por el SSN en el periodo 1998-2017, se observó que la sismicidad en la zona norte es la más baja en Veracruz y consiste únicamente de eventos corticales (no se observan sismos profundos). Además, por tratarse de una zona localizada en el sector oriental de la FVTM (región trasarco, Figura 2), el peligro asociado a los sismos interplaca que se originan en las costas del Pacífico de México es bajo, considerando la distancia a la fuente de los grandes eventos de este tipo. La situación con los sismos intraplaca es similar al caso anterior.

La zona central de Veracruz forma parte del sector oriental de la FVTM, por lo que se encuentra influenciada de una manera importante por los procesos tectónicos, sísmicos y volcánicos asociados a la presencia de ésta. En esta área tuvo lugar el sismo histórico más importante de la región, ocurrido el 4 de enero de 1920 (UTC), uno de los que más muertes ocasionó el siglo pasado en México, conocido como el sismo de Xalapa y cuya magnitud se estimó en Mw 6.4, con H 10 km y fallamiento normal (Suárez, 1992). Aún en la zona centro, al sur de la FVTM, tuvo lugar otro sismo histórico de la región: el sismo de Orizaba del 23 de agosto de 1973 (Figura 2), con Mw 7.1 a una profundidad intermedia de 80 km (Singh y Wyss, 1976). Este evento evidenció que la zona central de Veracruz también está expuesta a sismos importantes del tipo intraplaca, al igual que el ocurrido en 1937 (Figura 2) cercano a las localidades Acultzingo-Maltrata (M 7.3 y H = 85 km de acuerdo con Jiménez y Ponce, 1978).

Para el sur de Veracruz, la sismicidad en general que se ha reportado por el SSN incluye desde eventos corticales intraplaca, hasta sismos con foco en la placa subducida de profundidad intermedia y mayor (hasta 200 km). En esta región (ubicada en el antearco, Figura 2) tuvo lugar el sismo histórico del 26 de agosto de 1959 (Figura 2), conocido como el sismo de Jaltipan y que produjo severos daños en esta localidad y sus alrededores. Este evento tuvo una Mw 6.4 (con epicentro en el Golfo de México) y una profundidad $15 \mathrm{~km}$ con un régimen de esfuerzos de compresión (fallamiento inverso) según lo reportado por Suárez (2000). Otro 


\section{Observatorios - Observatorio Sismológico y Vulcanológico}

rasgo importante a destacar en esta zona es la presencia del Campo Volcánico Los Tuxtlas (CVLT, Figuras 2, 4 y 5), donde además de encontrarse uno de los volcanes considerado como activo en México, el San Martín Tuxtla, cuya última erupción data de 1793, se localizan más de 200 aparatos volcánicos (maars, conos de escoria, etc.) en un macizo rocoso inmerso en la cuenca oceánica de Veracruz. Además, dada la relativa cercanía de esta región a la zona de convergencia de las placas tectónicas Cocos y Norteamérica, existe un peligro sísmico importante que debe ser considerado y evaluado.

Por último, en el Golfo de México se han observado una serie de eventos (Suárez, 2000; Franco et al., 2013) aunque de magnitud moderada, deben tomarse en cuenta por su cercanía a centros poblacionales importantes tales como la ciudad de Veracruz, Minatitlán y Coatzacoalcos, entre otros. Algunos de ellos tienen su origen en la tectónica salina presente en esa área (Suárez, 2000; Franco et al., 2013).

\section{Reportes de sismicidad semestral}

El objetivo de los boletines que se proponen es primordialmente informar sobre los sismos que impactan al estado de Veracruz. Mediante una interpretación sismotectónica adecuada al contexto local, se pretende dar una comprensión de los fenómenos sísmicos que contribuya a la descripción del origen y comportamiento de los sismos en el área de interés, así como a la identificación paulatina de fallas activas y la evaluación del peligro sísmico local. Aunado a lo anterior, se pretende dar seguimiento a los propósitos de divulgación inherentes al Observatorio Sismológico y Vulcanológico de Veracruz (OSV) de acuerdo con su Plan Estratégico.

El Boletín, ver el Anexo 1, será publicado semestralmente en la página web del Observatorio Sismológico y Vulcanológico de Veracruz (https://www.uv.mx/osv/). Su contenido y elaboración se explica a continuación.

\subsection{Metodología}

Los eventos que se analizan corresponden a la sismicidad local en Veracruz reportada por el SSN durante el primer semestre de 2020. Estos eventos se seleccionaron del catálogo del SSN a partir de la especificación de un área rectangular en la página web www2.ssn.unam.mx:8080/catalogo/. Por medio de un Sistema de Información Geográfica (SIG, QGIS), los sismos obtenidos del catálogo se filtran para mantener aquellos cuyos epicentros se localizan dentro de los límites del estado de Veracruz. Los ocurridos en el Golfo 
de México (mar adentro) también se agregan. Con los programas GMT y QGIS se generan los mapas de sismicidad general del periodo elegido (Figura 4).
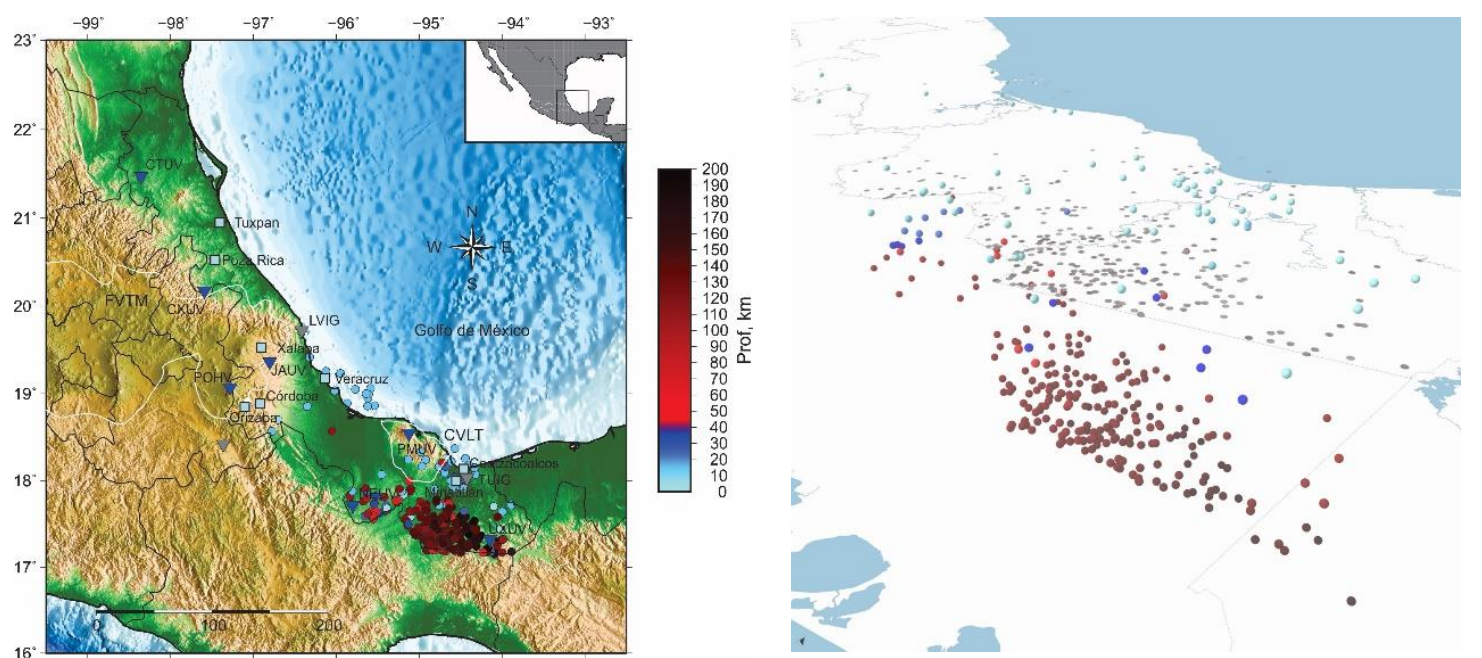

Figura 4. Mapa de sismicidad general en Veracruz reportada por el SSN para el periodo comprendido del 1 de enero al 30 de junio de 2020. (a) Vista en planta. (b) Representación en 3-D. Los círculos azules representan a los sismos ocurridos en la corteza continental, en tanto que los rojos corresponden a los localizados en la corteza oceánica. Los triángulos invertidos en color azul y gris señalan la ubicación en el estado de Veracruz de las estaciones de la Red Sísmica de Banda de Veracruz (RSBAV, Córdoba et al., 2018) y las del SSN respectivamente. Los cuadrados en azul, señalan la localización de las principales ciudades de la entidad veracruzana.

Los hipocentros de los sismos en Veracruz ocurren tanto en la corteza continental (eventos corticales), es decir en la placa Norteamérica, como en la placa oceánica subducida (placa Cocos, eventos intraplaca). Dado que el espesor de la corteza continental en el área de interés varía aproximadamente entre 30 y $40 \mathrm{~km}$, es posible considerar aquellos eventos con profundidad menor a $40 \mathrm{~km}$, como sismos corticales (placa Norteamérica); mientras los que se encuentran a profundidades mayores que este umbral, se asocian con la profundidad y la geometría de la placa de Cocos subducida. Como resultado de esta diferenciación, la Figura 5 muestran la clasificación de la sismicidad de acuerdo con la placa donde se originan los eventos. 

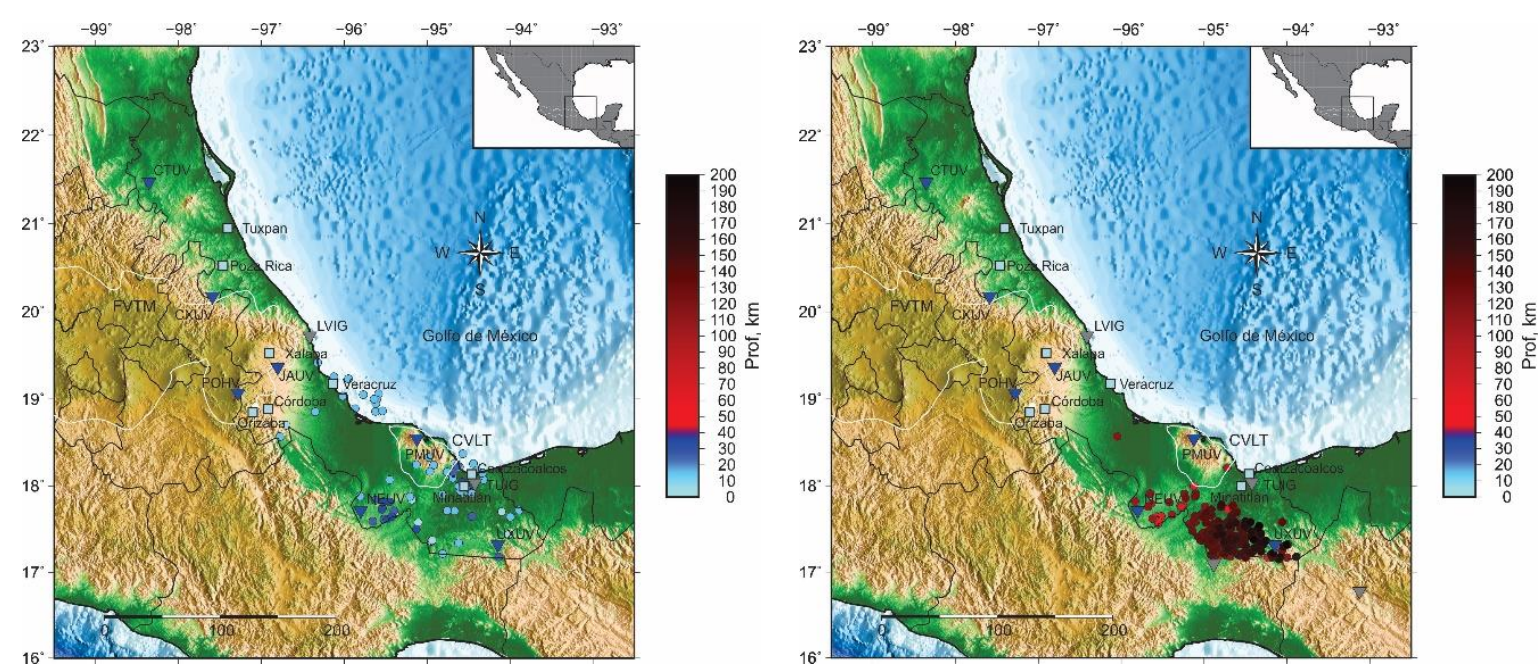

Figura 5. Mapas de sismicidad obtenidos asumiendo $\mathrm{H}=40 \mathrm{~km}$ como el umbral entre la sismicidad cortical (izquierda) e intraplaca (derecha) en Veracruz. Los triángulos invertidos en color representan las estaciones de la Red Sísmica de Banda Ancha de Veracruz (RSBAV, Córdoba et al., 2018).

Se incluye una gráfica que ilustra la variación temporal de la magnitud de la sismicidad reportada (Figura 6, izquierda). Se distinguen los sismos con $\mathrm{M}<4.0$ y $\mathrm{M} \geq 4$.0. La finalidad de esta clasificación es crear una referencia para corroborar que efectivamente no existe un incremento real de la sismicidad con el tiempo (identificar tendencias temporales). Con la incorporación de nuevas estaciones sísmicas a la red del SSN, la capacidad de detección aumenta y por lo tanto, es posible reportar una mayor cantidad de eventos que previamente pasaban desapercibidos para la percepción humana.
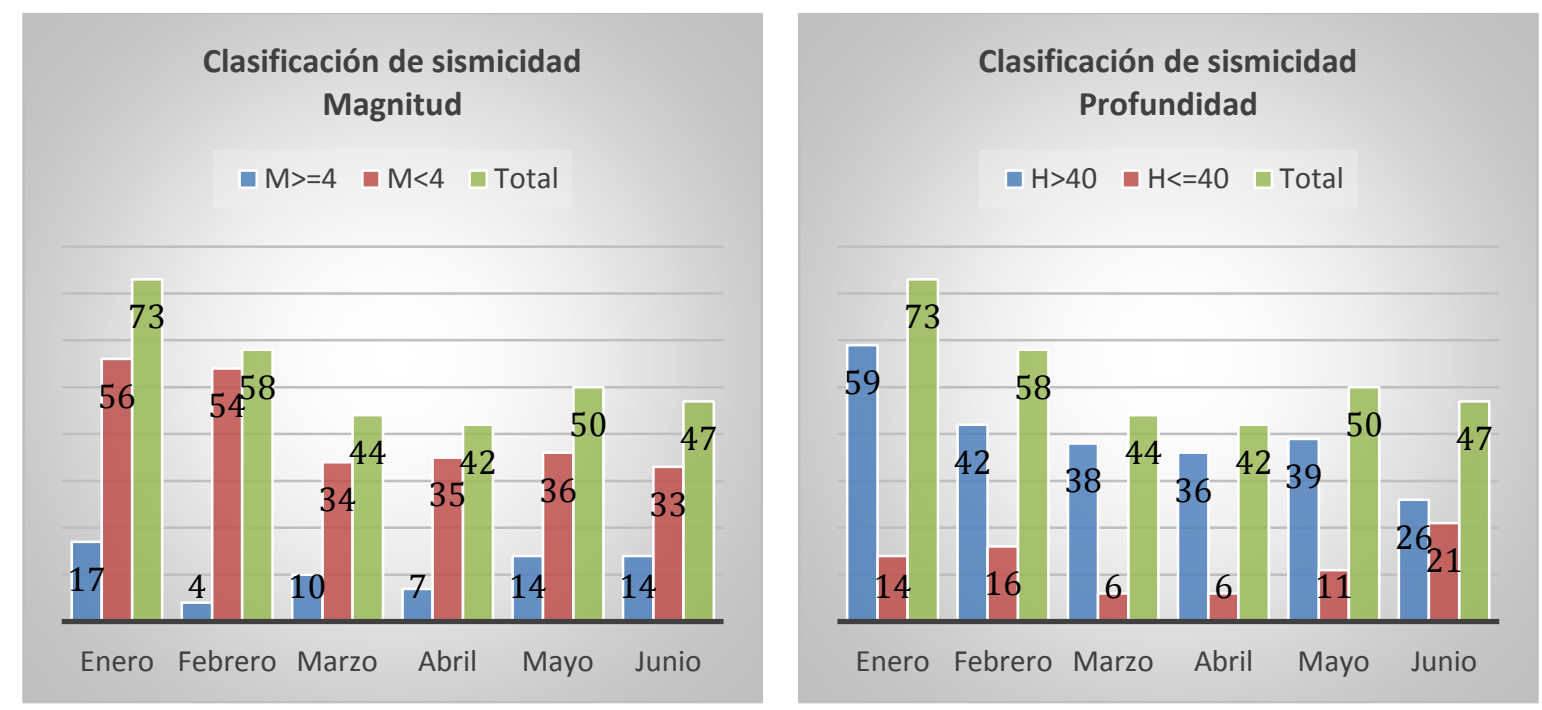

Figura 6. Gráficas de barras de variación de la sismicidad mensual de acuerdo con los umbrales de magnitud (izquierda) y profundidad (derecha) en Veracruz, reportada por el SSN, correspondiente al periodo comprendido del 1 de enero al 30 de junio de 2020. 
En la gráfica de la derecha en la Figura 6 se presenta la variación temporal de las profundidades de la actividad sísmica. Se distingue entre la sismicidad cortical y la sismicidad intraplaca. El valor umbral de profundidad propuesto es de $40 \mathrm{~km}$, aunque hay que recordar que el espesor de la corteza es variable. Esto implica en general que los sismos corticales cumplen con $\mathrm{H}<40 \mathrm{~km}$; mientras que los sismos intraplaca ocurren a profundidades mayores.

\subsection{Interpretación}

Finalmente, en la sección Interpretación Sísmica y Tectónica del Boletín, se realiza un breve análisis de lo observado en el periodo de evaluación a partir de la revisión de los mapas y gráficas que se generaron. En el caso de la sismicidad del semestre enero-junio 2020, esta interpretación es la siguiente. Aunque no se reportaron eventos en la zona norte de Veracruz, la sismicidad que se presenta en esa zona es solo cortical. En el caso de la zona centro, los eventos que ahí ocurren de acuerdo con el contexto tectónico son corticales e intraplaca. Sin embargo, no se reportaron sismos del segundo tipo $(\mathrm{H}>40 \mathrm{~km})$ para este período. En la zona sur de Veracruz, además de los sismos que se originan en la placa Cocos subducida, que da lugar a la principal sismicidad del área, y del estado de Veracruz en general, también se tiene el reporte de la mayoría de eventos corticales de la entidad (Córdoba et al., 2020).

\subsection{Publicación de boletines}

Por último, se diseñaron las diapositivas (como la que se muestra en la Figura 7) que anunciarán semestralmente la disponibilidad del nuevo boletín en la página Web del OSV. La diapositiva estará visible en la liga https://www.uv.mx/osv/. Todos los boletines históricos se almacenarán en la liga https://www.uv.mx/osv/boletines.

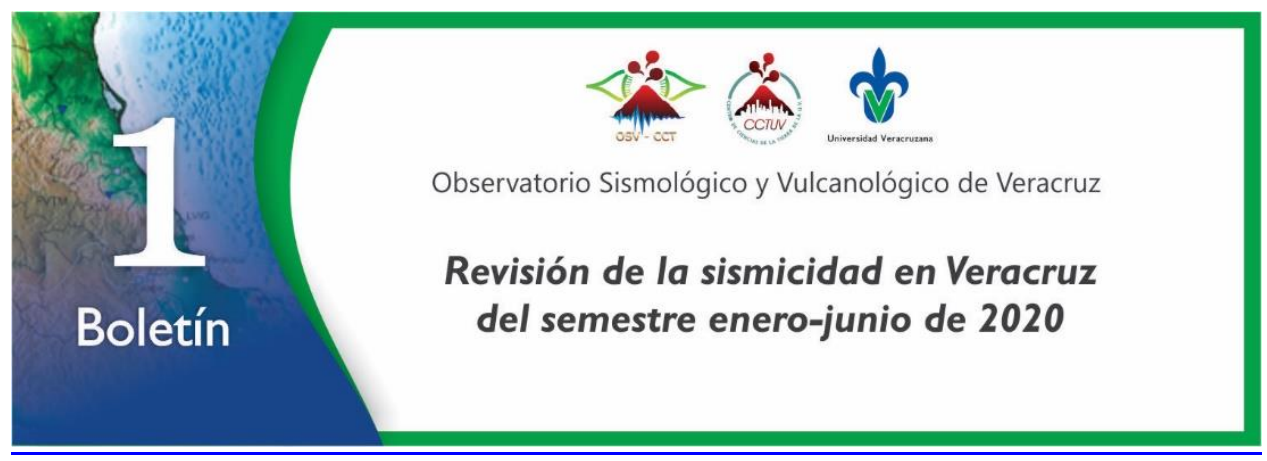

Figura 7. Ejemplo de diapositiva que se empleará para anunciar la disponibilidad en la página del OSV del boletín semestral más reciente. 


\section{Observatorios - Observatorio Sismológico y Vulcanológico}

\section{Conclusiones}

Aunque se considera que en Veracruz no suelen suceder sismos importantes, la historia sísmica de Veracruz indica la existencia de un peligro sísmico. La instalación y puesta en marcha de nuevas estaciones sísmicas ha permitido registrar una sismicidad a los procesos tectónicos de esta región del país. Para avanzar en la determinación del riesgo sísmico, se debe contar con una evaluación realista del peligro sísmico. El primer paso para lograr esto consiste en caracterizar la sismicidad base del área de interés. Esto implica conocer cuantitativa y cualitativamente los niveles de sismicidad y así poder establecer las condiciones locales que la originan. De esta manera se podrá avanzar en aspectos tales como la identificación de fallas activas, determinación del régimen de esfuerzos locales y regionales, propagación de ondas sísmicas, determinación de efectos de sitio, estructura cortical, y en general aquellos que contribuyan a la construcción del conocimiento necesario para determinar el peligro y riesgo sísmico.

\section{Reconocimientos y agradecimientos}

El catálogo de sismos del Servicio Sismológico Nacional (México) es posible gracias a todo su personal y producto de los cálculos realizados por su área de Análisis e Interpretación de Datos Sísmicos. Agradecemos el apoyo otorgado para la elaboración de este trabajo por parte del Proyecto CONACyT No. 255598.

\section{Referencias}

CFE (2008). Manual de Diseño de Obras Civiles. Diseño por Sismo, Comisión Federal de Electricidad. Instituto de Investigaciones Eléctricas.

Córdoba-Montiel, F., Iglesias A., Pérez-Campos X., Sieron K., Grupo de Trabajo del Servicio Sismológico Nacional, Valdés-González C., Singh S. K., Pacheco J. F., 2018. The Broadband Seismological Network of Veracruz, Mexico: towards a regional seismotectonic interpretation, Seism. Res. Lett., 89, 345-355. doi:10.1785/0220170195

Franco S.I., Canet C., Iglesias A., Valdés-González C., 2013, Seismic activity in the Gulf of Mexico. A preliminary analysis. Boletín de la Sociedad Geológica Mexicana, 65, 447-455.

Jiménez, Z., Ponce L., 1978. Focal mechanism of six large earthquakes in northern Oaxaca, Mexico, of the period 1928-1973. Geofísica Internacional, 17, 379-386.

MASE, 2007. Meso America Subduction Experiment. Caltech. Dataset. doi:10.7909/C3RN35SP. 
Melgar, D. \& Pérez-Campos, X., 2011. Imaging the Moho and subducted oceanic crust at the Isthmus of Tehuantepec, Mexico, from receiver functions. Pure appl. Geophys., 168(89), 1449-1460.

México. Universidad Nacional Autónoma de México, Instituto de Geofísica, Servicio Sismológico Nacional (2020), Catálogo de sismos, editado, UNAM, IGEF, SSN, doi:10.21766/SSNMX/EC/MX.

Pardo, M., Suárez, G. (1995), Shape of the subducted Rivera and Cocos plates in southern Mexico, seismic and tectonic implications, J. Geophys. Res., 100, 12357-12373.

Pérez-Campos, X. et al., 2008. Horizontal subduction and truncation of the Cocos Plate beneath central Mexico. Geophys. Res. Lett., 35(18), L18303

Singh S. K., A. Iglesias, M. Ordaz, X. Pérez-Campos, and L. Quintanar (2011). Estimation of Ground Motion in Mexico City from a Repeat of the M 7.0 Acambay Earthquake of 1912, Bull. Seismol. Soc. Am. 101 2015-2028.

Singh, S. K., Ordaz M., Pacheco J. F., Quaas R., Alcántara L., Alcocer S., Gutiérrez C., Meli R., Ovando E., 1999. A preliminary report on the Tehuacán México earthquake of June 15, 1999 (Mw = 7.0). Seism. Res. Lett., 70, 489-504.

Singh S. K., Wyss M., 1976. Source parameters of the Orizaba earthquake of August 28, 1973. Geofísica lnternacional, 16, 165-184.

Suárez G., 1992. El sismo de Jalapa del 3 de enero de 1920. Rev. Mex. de Ing. Sísmica, 42, 3-15.

Suárez, G. (2000). Reverse faulting in the Isthmus of Tehuantepec: Backarc deformation induced by the subduction of the Tehuantepec ridge, in Delgado, H., Aguirre, G., Stock,

J., (eds), Cenozoic Tectonics and Volcanism of Mexico, Spec. Pap. Geol. Soc. Am., 334, 263-268.

VEOX, 2010. Veracruz-Oaxaca Subduction Experiment. Caltech. Dataset. doi:10.7909/C3MW2F2C.

Yamamoto J., Jiménez Z., Mota R., 1984. El temblor de Huajuapan de León, Oaxaca, México, del 24 de octubre de 1980. Geofísica Internacional, 23, 83-110. 


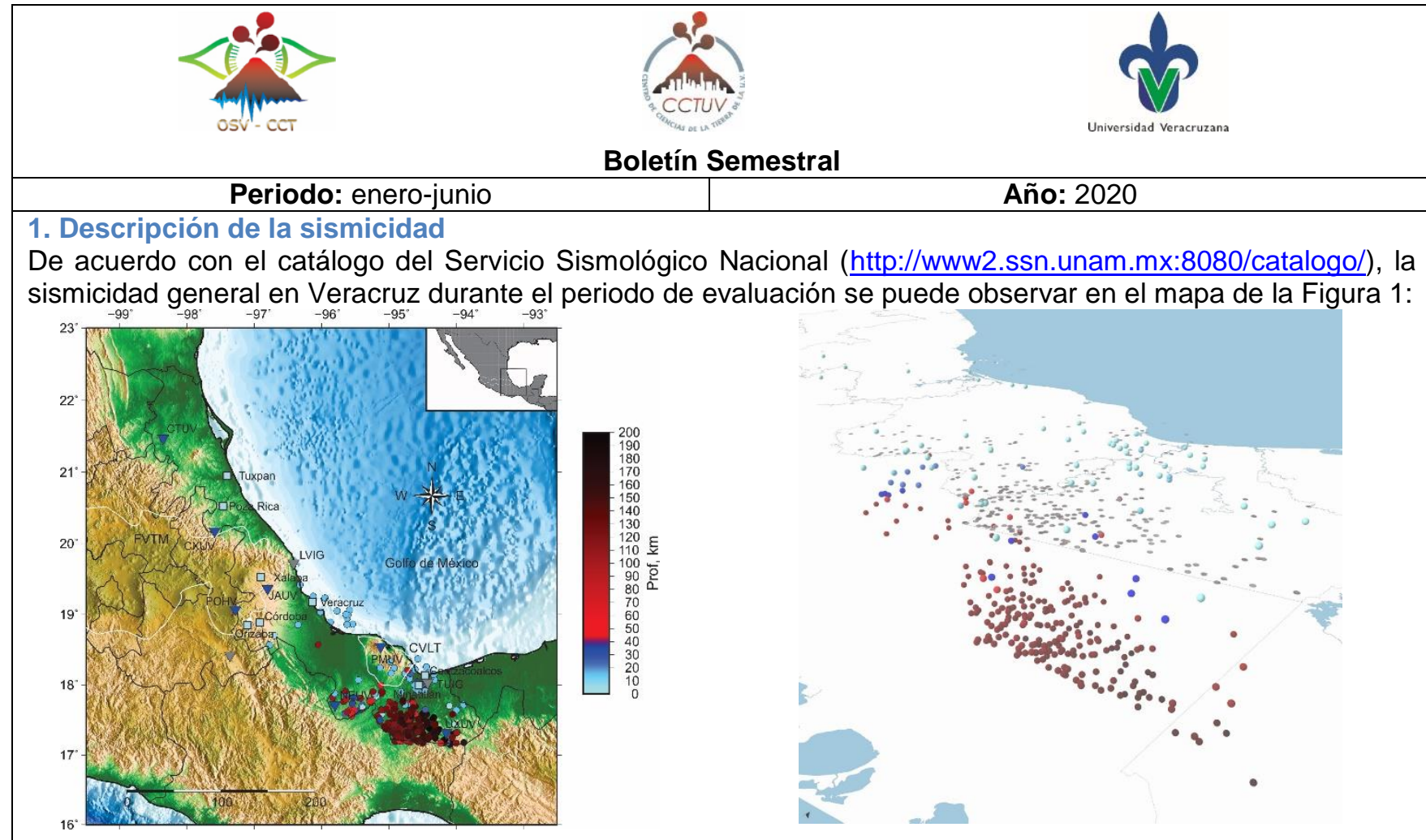

Figura 1. Mapa de sismicidad general en Veracruz reportada por el SSN para el periodo comprendido del 1 de enero al 30 de junio de 2020. (a) Vista en planta. (b) Representación en 3-D. Los círculos azules representan a los sismos ocurridos en la corteza continental, en tanto que los rojos corresponden a los localizados en la corteza oceánica. Los triángulos invertidos en color azul y gris señalan la ubicación en el estado de Veracruz de las estaciones de la Red Sísmica de Banda de Veracruz (RSBAV, Córdoba et al., 2018) y las del SSN respectivamente. Los cuadrados en azul, señalan la localización de las principales ciudades de la entidad veracruzana.

La sismicidad general de este periodo consistió en 314 sismos que de acuerdo con lo mostrado en la Figura 1, para la zona norte no se reportó ningún evento. En la zona centro se observa una escasa actividad sísmica, misma que se concentró particularmente en el Golfo de México frente a las costas de Veracruz-Alvarado. La mayor cantidad y densidad de eventos ocurrió en la zona sur del territorio veracruzano, principalmente en los límites con el estado de Oaxaca (Córdoba et al., 2020).
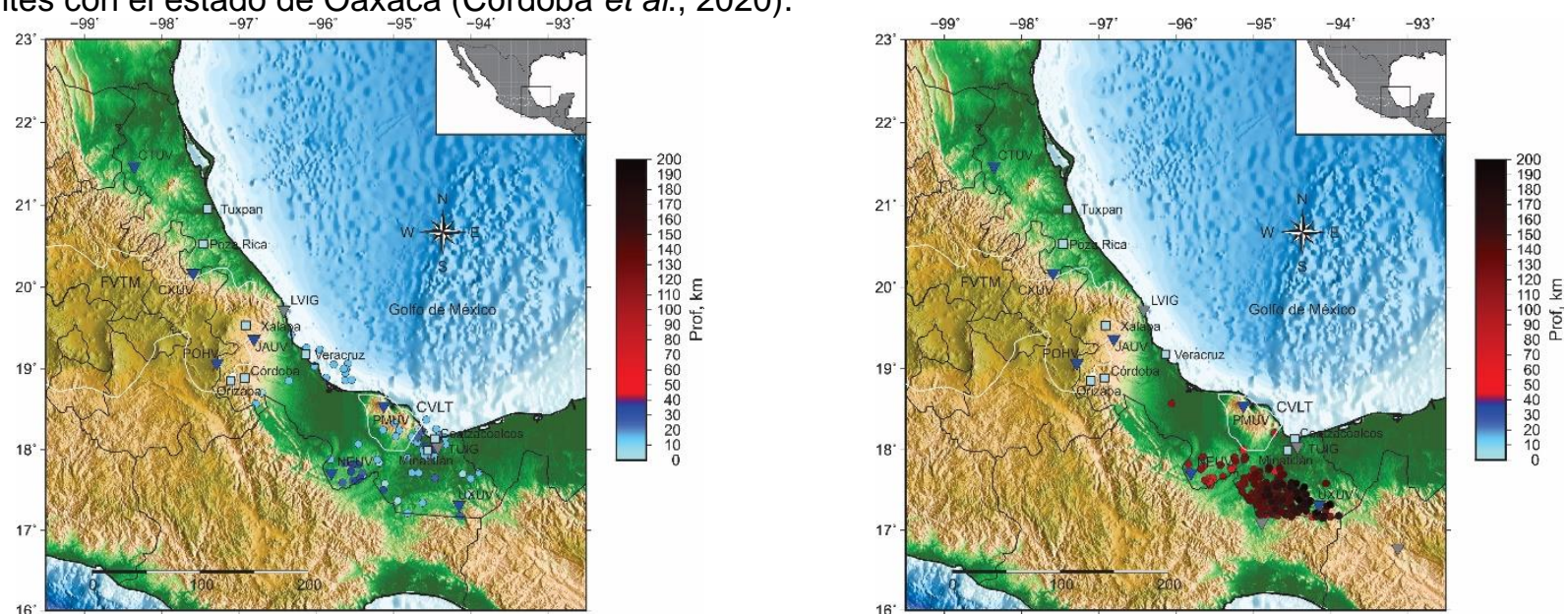

Figura 2. Mapa de sismicidad cortical (izquierda) y la sismicidad intraplaca (derecha) en el estado Veracruz reportada por el SSN para el periodo comprendido del 1 de enero al 30 de junio de 2020. 
En lo que respecta a la sismicidad de acuerdo con su origen, durante el periodo revisado de los 314 sismos, el número de eventos corticales (Figura 2, izquierda) es 74, de los cuales se distinguen dos grupos que se localizan en las costas de Veracruz y en la región sur de Veracruz. En el caso de los eventos intraplaca (Figura 2, derecha), se registraron en el periodo un total de 240 sismos, caracterizándose por su alta densidad en el límite con el estado de Oaxaca y sus profundidades mayores (Córdoba et al., 2020).
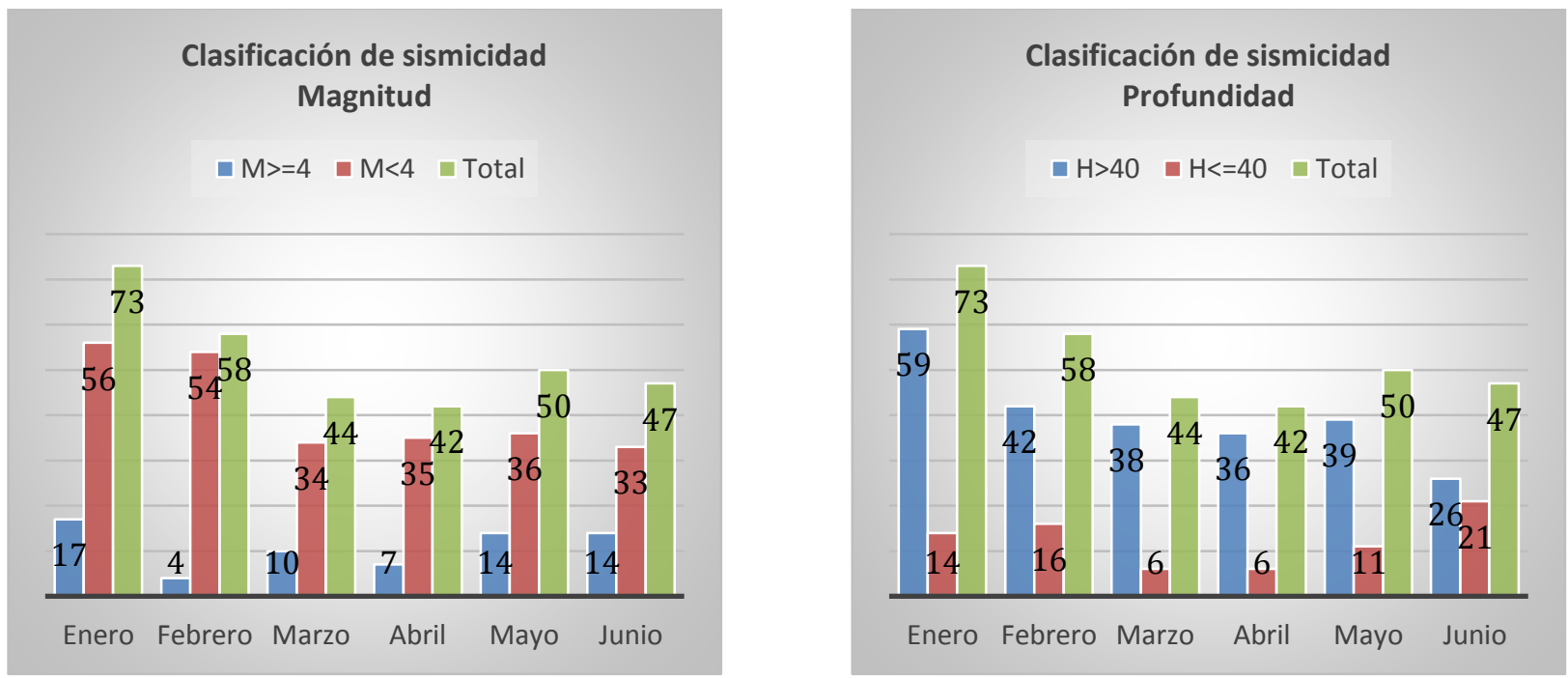

Figura 3. Variación mensual de la sismicidad de acuerdo con los valores de los umbrales de magnitud y profundidad propuestos por Córdoba et al., 2020.

La gráfica de la variación de la magnitud mensual (Figura 3, izquierda) indica que el número de eventos $M \geq 4.0$ es 66, de los cuales, el mayor tuvo una M 4.5. El promedio de estos eventos es 11 al mes, mientras que el promedio de sismos $\mathrm{M}<4$ es $\sim 41$. En el caso de la variación mensual los sismos corticales e intraplaca (Figura 3 , derecha), se reporta un promedio de 12 y 40 eventos respectivamente, lo que implica que solo $1 / 3$ de la sismicidad general ocurrió en corteza océanica subducida.

\section{Interpretación Sísmica y Tectónica}

Aunque no se reportaron eventos en la zona norte de Veracruz, la sismicidad que se presenta en esa zona es solo cortical. En el caso de la zona centro, los eventos que ahí ocurren de acuerdo al contexto tectónico son corticales e intraplaca. Sin embargo, no se reportaron sismos del segundo tipo $(\mathrm{H}>40 \mathrm{~km})$. En la zona sur de Veracruz, además de los sismos que se originan en la placa Cocos subducida, que da lugar a la principal sismicidad del área y del estado de Veracruz en general, también se tiene el reporte de la mayoría de eventos corticales de la entidad (Córdoba et al., 2020).

\section{Reconocimientos y agradecimientos}

El catálogo de sismos del Servicio Sismológico Nacional (México) es posible gracias a todo su personal y producto de los cálculos realizados por su área de Análisis e Interpretación de Datos Sísmicos.

La Red Sísmica de Banda Ancha de Veracruz opera desde 2013 gracias a la colaboración tripartita entre la Universidad Veracruzana, el Servicio Sismológico Nacional y la Secretaría de Protección Civil de Veracruz.

\section{Referencias}

Córdoba-Montiel F., X. Pérez-Campos, K. Sieron, S.F. Juárez Cerrillo, Grupo de Trabajo del Servicio Sismológico Nacional, Coordinación Universitaria de Observatorios (2020). Análisis e interpretación de la sismicidad local en Veracruz para la generación de boletines del OSV. UVserva 10.

México. Universidad Nacional Autónoma de México, Instituto de Geofísica, Servicio Sismológico Nacional (2020), Catálogo de sismos, editado, UNAM, IGEF, SSN, doi:10.21766/SSNMX/EC/MX. 